\title{
Perinatal Assessment of Risk of Mental Illness: Experience of First-time Mothers and Clinicians, Australian Private Hospitals
}

Deborah Jane Sims ( $\nabla$ debsims@bigpond.com )

University of Technology Sydney https://orcid.org/0000-0002-8340-5083

Cathrine Fowler

University of Technology Sydney

Christine Catling

University of Technology Sydney

Fenglian Xu

University of Technology Sydney

Research article

Keywords: Mental illness, psychosocial assessment, perinatal, private hospital, experience

Posted Date: September 23rd, 2019

DOI: https://doi.org/10.21203/rs.2.14832/v1

License: (c) (i) This work is licensed under a Creative Commons Attribution 4.0 International License.

Read Full License 


\section{Abstract}

Background The many changes in becoming a mother increase the risk of developing a mental illness in the perinatal period. Comprehensive, psychosocial assessment for risk of mental illness is recommended as part of routine perinatal care for all women. In Australia, this assessment was less likely to be undertaken for women who gave birth in a private hospital compared to women who gave birth in a public hospital. Therefore this study aimed to explore the experience of perinatal assessment of risk of mental illness for first-time mothers who gave birth in a private hospital. Method The results are from an explanatory mixed-methods study on the risk of the development of a perinatal mental illness and its relationship with the delivery of, or lack thereof, a parenting support service. Interviews were conducted with first-time mothers, midwives, nurses, obstetricians and paediatricians at two Australian metropolitan private hospitals. Content analysis of the interview data provided exploration and interpret their experience of assessment of risk of mental illness. Results Interview data were gathered from 32 participants. Four themes on assessment of risk of mental illness for these women were described: part of routine perinatal care; comprehensive psychosocial assessment; coordination between care providers and lack of mental health resources. Conclusion For mothers who gave birth in a private hospital, comprehensive psychosocial assessment of risk of mental illness may not have been provided as part of the routine perinatal care and not coordinated between care providers. A lack of mental health resources cannot be regarded as a barrier to provision of these services.

\section{Background}

The many changes experienced while becoming a mother, increase the risk of developing a mental illness $[1-4]$. One in five women will experience mental health issues during the perinatal period [4, 5]. Mental illness during the perinatal period may have serious long-term effects on the wellbeing of women, their babies and families [6-9]. During pregnancy the complications of mental illness are reported to be higher than physical complications such as gestational diabetes and hypertension [10]. Despite a quarter of women experiencing perinatal mental illness developing chronic or recurrent illness two thirds will remain untreated [11].

Guidelines that aim to improve this deficit recommend a program of assessment of the risk of mental illness for all women that: is integrated within routine care, provided as a comprehensive, psychosocial assessment; is integrated within perinatal care; and is not withheld due to lack of resources [12-17]. However, women who gave birth in an Australian private hospital were less likely to be provided with assessment of their risk of developing mental illness $[18,19]$. National guidelines recommend that both protective and risk factors for mental illness are assessed in the perinatal period within the context of the parenting role, as an integral component of assessing the entire parenting and integration of assessment tools, such as the Edinburgh Postnatal Depression Scale, is part of effective psychosocial assessment [12]. Assessment tools provide a starting point and then allow comparison to detect change over time [15]. 
This study aimed to explore assessment of the risk of mental illness for mothers who gave birth in a private hospital through thematic analysis of the experiences of women and clinicians. Exploration and integration of interview data provided four themes on assessment: part of routine care, comprehensive psychosocial assessment, coordinated perinatal care and mental health resources.

\section{Methods}

Perinatal mental illness risk assessment was examined as part of an explanatory, sequential mixedmethods study on social support services, self-efficacy and risk of mental illness for first-time mothers who gave birth in a private hospital. A qualitative descriptive methodology provided the most flexible method to gather, explore, interpret and present findings on the experiences of first-time mothers and clinicians [20]. The research explored the experiences of diverse experiences in order to circumvent generalisability [21-23].

\section{Recruitment}

Participants were recruited from two private hospitals in metropolitan NSW, Australia. Both hospitals provided maternity care for a diverse multicultural population of 2000 women who give birth each year. Participants included both first-time mothers and clinicians. Primigravid women who had received maternity care in the hospital in the prior four to six months were recruited from both hospitals. Additionally, clinicians who provided maternity care were recruited from both private hospitals. Midwives, obstetricians, nursery nurses and paediatricians were invited to participate. These participants were recruited using both convenience sampling and snowball sampling [21, 24]. To maximise diversity of experience data multiple, simultaneous recruitment strategies were employed. These are listed in Box 1.

\section{Data Collection}

Following recruitment, data were collected through interviews and two focus group interviews. An important aspect of data collection and analysis was the verification cycle of questioning and confirmation that was undertaken at each stage: during interviews, between interviews and between phases. This process narrowed the field of endeavour and thus provided focus that allowed the collection of diverse data on the assessment of risk of mental illness [25].

Midwives and nurses were invited to participate in focus-groups that encouraged discussion between participants and facilitated a variety of perspectives [23]. To promote the likelihood of discussion occurring, focus group questions were piloted on a group of nurses and midwives who were not participating in the study. Obstetricians, paediatricians and first-time mother participants were invited to participate in semi-structured interviews. The interview questions for clinicians were piloted on a paediatrician and an obstetrician who were not participating in the study. The interview questions for firsttime mothers were piloted on two first-time mothers who were not participating in the study. This 
refinement of questions ensured that they were focused on the purpose of the study, being the risk assessment of mental illness, and as a consequence, the interview process minimised any likelihood of a negative effect on the participant, especially on the mother-baby dyad. Given this consideration, interview questions are provided in the supplementary information.

Analysis of the data was undertaken in two distinct steps. Analysis Step 1 began as interviews were conducted and the researcher reviewed transcripts to discover additional questions or offer additional findings [25]. Step 2 was thematic analysis of assessment of the risk of the development of mental illness based on the literature. Box 2 defines the four subthemes.

\section{Results}

Interviews were conducted with eight first-time mothers aged in their 30 s, six of whom had completed tertiary education. Five of the babies were male and three were female. Twenty three clinicians contributed; 11 midwives, three nurses, six obstetricians and three paediatricians. The demographic characteristics of these participants are provided in (Tables 1 and 2).

The findings encapsulated the stakeholder experience of perinatal risk assessment of mental illness for first-time mothers who had given birth in a private hospital. The four themes were 1) part of routine care, 2) a comprehensive psychosocial assessment 3) integrated care 4) mental health resources.

Pseudonyms have been used to maintain anonymity.

\section{Part of Routine Care}

In both focus groups, the midwives and nurses agreed with each other that perinatal psychosocial assessment was not routinely provided for women who gave birth in that hospital. Additionally, focus group 1 participants concurred that assessment was part of routine care for women who gave birth in a public hospital:

Participant 2:I think a lot of that [responsibility] sort of goes on the obstetricians, in terms of antenatally. We often don't meet them [mothers] until they're in hospital, or maybe a class. But in the public system they do.

Participant 3:That's probably handled a lot better in the public system.

This quote demonstrated the midwives' and nurses' belief that assessment was not provided as part of routine care for women as well as their belief that assessment was provided for women who gave birth in the public hospital system. These understandings were supported by all participants within both focus groups who demonstrated agreement, either verbally or through body language (as noted within field note entries). 
While psychosocial assessment is strongly advised as part of routine care for mothers it is not consistently implemented. The nurses and midwives went on to explain that some obstetricians who provided care at their hospital do organise a referral for screening for all of mothers for whom they provided care.

Participant 1:Some of our obstetricians do refer all their ladies to clinical psychologist to do screening during pregnancy.

Participant 2: But not all do.

(Field note: All other participants gestured agreement, verbally or head nod.)

The agreement among participants demonstrated their belief that risk assessment of mental illness was provided as part of routine care for only some women. This assessment was determined by which obstetrician was providing care.

During interviews the women were asked if they had received assessment of their emotional health and well-being or of their mental health during the perinatal period. The following response was typical:

Interviewer: So, in regard to your emotional health and well-being, or your mental health, did you have any formal assessment during the pregnancy?

Jessica: No? (answered with puzzled expression on face and questioning tone in voice)

Interviewer: What about after the pregnancy?

Jessica: No? (answered with puzzlement)

Jessica's response was clear that she had no recollection of assessment, either prenatally or postnatally. In addition, the puzzled expression on her face and her questioning tone of voice when she answered 'No?' conveyed that assessment was unfamiliar. Five of the eight interviewed did not recall undertaking any assessment of emotional well-being or mental health.

One of the women who had received an assessment, explained her surprised that all women did not receive assessment:

I've also heard that not all obstetricians do that assessment. When I've mentioned to a few of my friends that doctor [name] had got me to do the [Edinburgh Postnatal Depression Scale] questionnaire, they were quite shocked and said that not all obstetricians do that. (Rebecca)

Rebecca may have thought that the Edinburgh Postnatal Depression Scale was routine care for all women. Her experience that other mothers were shocked that she had completed the EPDS demonstrated that other women were unfamiliar with the assessment. 


\section{Comprehensive Psychosocial Assessment}

Three of the women did receive assessment; although not a comprehensive psychosocial assessment, there was variety in the assessment content. Postnatal mental health was informally assessed for one mother through the clinician asking a question. Lauren's family doctor asked her about coping?

... she [family doctor] always asks if I'm okay? Especially because he [baby Logan] has had a lot of issues, [she asks] how am I mentally coping? (Lauren)

The assessment approach used by this family doctor is limited to a response to a single question. A similar approach was undertaken by one of the obstetricians, who assessed mental health of the mother as part of routine postnatal care, using a single question on the woman's mood:

I believe I have a better feel for the patient [mental health] if I ask them directly how their mood has been. It is one of my standard questions in the six-week postnatal review. But I don't use a standardised tool. (Obstetrician 004)

The midwives and nurses in both focus groups were asked if a tool was used to assess mental health. This participant confirmed that midwives did not use a tool for the assessment, but she believed that midwives should use a tool to assess risk of mental illness by stating:

Participant 1:I think we should, but we don't.

In this group the participants agreed, either using a verbal affirmative or through body language. The participants' response was the same in the second focus group, suggesting that at these two hospitals midwives and nurses do not use tools to assess risk.

In another example Nicole explained that the obstetrician provided an Edinburgh Postnatal Depression Scale. She explained how easily she manipulated the screening tool and pretended that she was well in order to avoid referral to as psychologist. In this way Nicole was able to manipulate her responses and avoid referral for assistance.

So, if you don't want to see someone, it's very easy to answer them, so that you don't have a problem and I don't know if that's a common thing with people with mental health but I mean, I know that I had a psychologist that I didn't like, so for three sessions in a row I said I was fine. ... those tests are so easy, it's not a secret test, you pick the right answer and you get a good score. So, if you don't want help, or if you don't want to admit you've got a problem, then it's very easy to avoid it. (Nicole)

This description from Nicole showed how tailoring Edinburgh Postnatal Depression Scale responses either enables access to psychological support or to avoid this potential diagnosis of mental illness and subsequent support.

\section{Coordinated Between Care Providers}


Coordination of assessments of risk of mental illness between care providers was not evident in the findings. Some of the women recalled being assessed by different clinicians at different times. Nicole recalled that in the postnatal weeks she completed one EPDS with her obstetrician and on multiple occasions, with a child and family health nurse.

... my obstetrician, she did one [Edinburgh Postnatal Depression Scale]. Um, I scored badly. I then did one [Edinburgh Postnatal Depression Scale] with the family health woman [nurse]. I scored worse. [Laughs] 21 out of 30 or something, on the Edinburgh thing [Edinburgh Postnatal Depression Scale]. I then did another one, because she [nurse] likes to keep up on that, and I needed to get another referral so that I could see my psychologist. And no doubt, I will probably end up having to do another one again. (Nicole)

Her tone of voice and turn of phrase suggested that she appeared resigned to the inevitability of completing another Edinburgh Postnatal Depression Scale in order to obtain a referral to a psychologist and of having to complete further Edinburgh Postnatal Depression Scale in the future. Such an experience suggests a lack of integration of postnatal care between the obstetrician and the nurse.

\section{Mental Health Resources and Assessment}

The clinicians provided women with information to access support for mental illness when they recognised a need. The following examples were typical of the support organisations for women experiencing perinatal emotional distress or illness that clinicians described. This midwife listed three mental health resources that she recommends to all women in her care.

So, Internet wise, I talk [to the women] about PANDA, I talk about Beyond Blue, I talk about Gidget Foundation, as they all have great online resources. (SF002)

All of the midwives and nurses in the focus group indicated their agreement, through their body language (nodded their heads) or verbally ('yes'). In the other focus group the same resources were listed by one participant and the other participants confirmed agreement. Paediatricians and obstetricians also provided women with access to support for mental illness

One of the paediatricians discussed facilitating access to mental health resources. In this example the paediatrician accessed a support service on the woman's behalf.

I've had a few patients who have had significant psychiatric problems and if I feel they're not coping I definitely speak to the obstetricians. We've accessed in-home care for some of those families [where the woman has significant psychiatric problems]. (NP003)

Although no formal assessment of mental illness was undertaken, an awareness of mental illness prompted the paediatrician to access in-home support services. Obstetricians also facilitated access to mental health resources. 
In this example the woman explained that the obstetrician accessed a support service on her behalf, after she completed an Edinburgh Postnatal Depression Scale. Her obstetrician referred her to her family doctor to have a mental health plan developed that would allow access to some additional resources.

... he [family doctor] had to do a whole mental health assessment thing. (Rebecca)

The obstetrician assessed the risk of mental illness using the Edinburgh Postnatal Depression Scale, which prompted a referral to the woman's family doctor to access support services. This suggests that the obstetrician was aware of how the mother may be able to access the additional services through community services, yet did not undertake the mental health plan, possibly due to time constraints or experience.

\section{Another woman recalled a prenatal assessment at the hospital that led to support from a psychologist:}

I had one [emotional health assessment] just before Eli was born ... they offered it as part of the [hospital] service and I saw a psychologist at the hospital and she was great. (Amanda)

Amanda went on to describe how the support assisted her with the transition to parenthood.

She just helped me talk about the differences there were going to be from going ... from quite a big job that I have ... into being a mum. You know, a big change, and taking time off, and how things will be hard. And she was really good at that and making sure that I knew what was my purpose and I defined myself, so I didn't get lost in myself. (Amanda)

Amanda has provided a thoughtful response that identifies the potential values for her of a one-off psychological intervention to provide encouragement for the development of protective intervention. Unfortunately mental health resources were only available to women, if the need was recognised by the clinician. This would suggest that a lack of resources was not a barrier to risk assessment as part of routine care, but required the active commitment of clinicians to facilitate access to these resources and support.

These findings demonstrated that in these private hospitals perinatal assessment of risk of mental illness was not provided as part of routine care for all women. When assessment was undertaken it was not as a comprehensive psychosocial assessment and there was a lack of coordination of assessment between hospital and community providers. A lack of mental health support services was not demonstrated as a barrier to undertaking assessment.

\section{Discussion}

There is limited research published into assessment of risk of mental illness for women who gave birth in a private hospital. The studies that have been published show that assessment was not provided as part 
of routine care. Studies on assessment of perinatal mental illness have determined that neither the Edinburgh Postnatal Depression Scale nor the use of a single interview question provide comprehensive psychosocial information. Studies on care across the perinatal period have demonstrate fragmentation of care between hospital and community providers.

\section{Part of Routine Care}

The findings from this new study indicate that the women were not provided with a comprehensive psychosocial assessment to identify the of risk of mental illness as part of their routine perinatal care. This concurred with a previous study that examined perinatal risk of mental illness and demonstrated that women who gave birth in a private hospital were less likely to undertake formal assessment [18]. Two studies of private hospitals that had implemented an assessment program demonstrated high levels of acceptability $[19,26]$. One of the focus group participants explained that as women are under the care of an obstetrician prenatally the midwives may be restricted in the assessment they can conduct.

\section{Comprehensive Psychosocial Assessment}

These findings indicated assessment was not undertaken as a comprehensive psychosocial assessment. Participants in this study recalled either being asked one question on coping or completing the Edinburgh Postnatal Depression Scale. Use of a single form of assessment allowed some women to avoid detection. Neither the women, clinician or administrator participants discussed comprehensive psychosocial assessment although guidelines recommend a comprehensive clinical psychosocial interview, which may include the use of a structured tool such as Edinburgh Postnatal Depression Scale $[17,27,28]$ to facilitate earliest identification of risk of mental illness. The aim of comprehensive psychosocial assessment is to provide a multi-dimensional picture of the mother's psychosocial circumstance, not just symptoms of a mental illness [27]. The recommendation is for inclusion of risk factors; inadequate social support, intimate partner relationship issues, isolation, stressful life events, personality vulnerabilities, poverty, history of trauma and history of mental illness or substance abuse [27]. An Australian study of a public hospital screening program identified that $69.1 \%$ of midwives screened for depression using Edinburgh Postnatal Depression Scale [29]. Edinburgh Postnatal Depression Scale is a valuable instrument to assist recognition of perinatal mental illness; it was originally validated by health visitors of 84 women, revealing sensitivity over time and judged as successful in increasing the delivery of both sensitivity and specificity when family members were not present [30]. A review of 36 Edinburgh Postnatal Depression Scale studies showed moderate consistency and accuracy [31]. Edinburgh Postnatal Depression Scale has been known to include ambiguity, exclude certain types of distress, have low positive predictive value, attempt to provide validation against a questionable gold-standard and misdiagnosis of transient distress; thus users of the scale should be aware that it, like other measures, has limitations. [32]. Appropriate care was shown to be problematic following administration of a screening tool at a single time point [33]. In a study of a residential EPS service, experienced child and family health nurses integrated information from assessment tools into 
psychosocial assessment when inconsistencies or mismatches exist in order to pinpoint or confirm areas of risk [34]. Mental health aspects are entwined within the parenting issues and cannot be isolated from them [35]. In particular, perinatal psychosocial risk factors include recent stressors, mental health history, lack of partner support and history of abuse. (Austin \& Committee 2013; Austin; Leigh \& Milgrom 2008; Milgrom et al. 2011; National 2007). In Australia, child and family health nurses have a comprehensive knowledge of perinatal mental health risk assessment within child health and parenting framework from specialist education [36]. Most importantly, in a study of residential early parenting services, the experienced child and family health nurses determined vulnerability to perinatal mental illness may compromise ability to parent and identify interventions that diminish the risk of developing a mental illness [34]. The completion of a questionnaire may decrease the likelihood that women will be truthful, where a comprehensive assessment of risk factors obtained through conversation may build rapport and increase trust, resulting in a more accurate assessment of risk as well as development of resources for the woman.

\section{Coordinated Perinatal Care}

The findings of this study describe that assessment may not be coordinated between hospital and community providers. This finding is supported by other studies of perinatal services that demonstrated fragmentation and lack of co-ordination between early parenting support services [37, 38]. Guidelines recommend that perinatal care is integrated between primary, secondary and tertiary health systems; across health disciplines; between assessment and referral; across the perinatal time periods; and between service settings [27]. An American study disclosed that obstetrician assessment was lacking in detection, treatment and referral [39]. Midwives were more likely than obstetricians to ask about mental health [40]. A qualitative study of perinatal psychosocial assessment described a negative emotional effect on the midwives from the cumulation of complex disclosure, frustration regarding referral and lack of support [41].

\section{Mental Health Resources}

The clinicians provided examples of actions taken when a women was identified as having potential risk factor. Importantly, a lack of resources or support service was not a barrier to assessment, as when clinicians recognised a need they did provide mothers with mental health resources. As this suggests that a lack of mental health resources was not the reason that perinatal assessment of risk of mental illness was not routinely provided to these women, the benefits of perinatal assessment are to provide information on factors that impact wellbeing and raise awareness on care options [27]. National guidelines suggest one outcome of psychosocial assessment may be referral to mental health services [28]. Additionally the Australian Longitudinal Study on Women's Health Women subsample (778) showed that women who were not asked about their emotional health were less likely to seek support during the perinatal period [42]. Perinatal assessment of risk of mental illness provides an opportunity for women to 
develop social supports, potentially both as an individual support service and a community support network, proving an effective mechanism that can ameliorate the risk of perinatal mental illness [27].

\section{Conclusion}

In line with international recommendations and Australian legislation, this study provided evidence that comprehensive psychosocial assessment of risk of mental illness was not provided as part of routine, integrated perinatal care for women who gave birth in private hospitals, although mental health support services being available.

\section{- Recommendations}

The comprehensive assessment of risk of mental illness as part of routine, coordinated perinatal care has been demonstrated to be feasible and highly acceptable in the private hospital setting $[26,43]$ and should be available to all women who give birth in a private hospital.

\section{Limitations}

The sampling strategy that was used for this study, snowball sampling, makes it impossible to make inferences about populations based on the obtained sample. Future studies may investigate the situation at other private hospitals in other settings.

In order to define the issue future studies may determine the prevalence of perinatal mental illness for women who gave birth in a private hospital compared to women who gave birth in a public hospital. A description of assessment experiences between new mothers who gave birth in a public hospital compared to first-time mothers who gave birth in a private hospital would provide greater depth on this topic.

Additionally, as paternal depression has been shown to correlate with maternal depression in $10 \%$ of partners during the perinatal period [44], further research that considers assessment experience of firsttime fathers may provide practical information to develop services for mothers who give birth in a private hospital.

Issues of debate on screening; sensitivity, specificity, positive predictive value and clinical cut-off remain outside the scope of this study.

- $\quad$ Clinical Practice Contribution

This evidence may strengthen implementation of services to provide comprehensive assessment of risk of mental illness as part of routine, coordinated perinatal care for women who give birth in a private hospital. Provision of this services may facilitate early intervention that has the potential to ameliorate the risk of mental illness and improve outcomes for women and their families. 


\section{Declarations}

- Ethical Approval-The study was approved by the NSW Population and Health Services Research Ethics Committee (AU RED Reference: HREC/11/CIPHS/33) on 13 May 2016; the Human Research Ethics Committee of the University of Technology Sydney, Australia (ETH16-0839) on 3 April 2017; North Shore Private Hospital Ethics Committee (NSPHEC2017-LNR-006) on 14 June 2017 and Sydney Adventist Hospital Ethics Committee (HREC2017-009) on 14 August 2017

- Consent to participate-all participants received ethics committee approved information and voluntarily agreed to consent to participate in writing.

- Consent for publication-Not Applicable

- Availability of Data and Materials - The datasets generated and analysed during this study are unavailable due to small sample size potentially allowing identification of participants.

- Competing Interests - The authors had neither financial nor non-financial competing interests.

- Funding - This research was supported by Australian Research Training Program (S93077814) and NHMRC Training Fellowship (Application ID 568892). The funding sources were not involved in the design of the study, the collection, analysis, and interpretation of data and in writing the manuscript.

- Author Contributions-DS was the main contributor through design, data; collection, analysis and interpretation. CF, CC and FX supervised the design of the study and provided guidance at each stage. CF was a major contributor providing assistance with analysis and interpretation of the data. All authors contributed to creation of the manuscript.

- Acknowledgements-Not Applicable

\section{References}

1.Maxson P, Edwards S, Valentiner E, Miranda M. A Multidimensional Approach to Characterizing Psychosocial Health During Pregnancy. Maternal \& Child Health Journal. 2016;20(6):1103-13.

2.Rasmussen M-LH, Strøm M, Wohlfahrt J, Videbech P, Melbye M. Risk, treatment duration, and recurrence risk of postpartum affective disorder in women with no prior psychiatric history: A population-based cohort study. PLOS Medicine. 2017;14(9):e1002392.

3.Woolhouse H, CGartland D, Mensah F, Brown SJ. Maternal depression from early pregnancy to 4 years postpartum in a prospective pregnancy cohort study: implications for primary health care. Royal college of obstetricians and gynecologists. 2014.

4.Epifanio MS, Genna V, De Luca C, Roccella M, La Grutta S. Paternal and maternal transition to parenthood: the risk of postpartum depression and parenting stress. Paediatric Reports. 2015;7(5872).

5.Milgrom J, AW. G. Screening for perinatal depression. Best

Pract. Res Clin Obstet Gynaecol 2014;28(1):13-23. 
6.Jarde A, Morais M, Kingston D, Giallo R, MacQueen G, Giglia L, et al. Neonatal outcomes in women with untreated antenatal depression compared with women without depression: a systematic review and meta-analysis JAMA Psychiatry. 2016;73(8):826-37.

7.Giallo R, Woolhouse H, Gartland D, Hiscock H, S. B. The emotional-behavioural functioning of children exposed to maternal depressive symptoms across pregnancy and early childhood: a prospective Australian pregnancy cohort study. Eur Child Adolesc Psychiatry. 2015;24:1233-44.

8.Murray L, Halligan SL, Goodyer I, Herbert J. Disturbances in early parenting of depressed mothers and cortisol secretion in offspring: a preliminary study. J Affect Disord. 2010;122(3):218-23.

9.Kerstis. association between depresison and impaired bonding. 2016.

10.Centre for Epidemiology and Evidence. New South Wales Mothers and Babies. Sydney, Australia; 2016.

11.Patten SB, Kennedy SH, RW. L. Canadian Network for Mood and Anxiety Treatments (CANMAT) clinical guidelines for the management of major depressive disorder in adults. Classification, burden and principles of management. Journal Affective Disorders. 2009;117 (Supplement 1):5-14.

12.Austin M-P, Highet N. Mental health care in the perinatal period: Australian clinical practice guideline. Melbourne, Australia: Centre of Perinatal Excellence; 2017.

13. Health. WAfIM. WAIMH Position Paper on the Rights of Infants. Edinburgh: World Association for Infant Mental Health; 2016 March 2016.

14. Health Do. Clinical Practice Guidelines: Pregnancy Care. Canberra: Australian Government Department of Health; 2018.

15.Gynecologists ACoOa. ACOG Committee Opinion No. 757. Screening for perinatal depression. Obstet Gynecol. 2018;132e:208-12.

16.Scottish Intercollegiate Guidelines Network (SIGN). Management of perinatal mood disorders. Edinburgh; 2012.

17.National Institute for Health and Clinical Excellence (NICE). Antenatal and postnatal mental health: The NICE guidelines on clinical management and service guidance CG45. National Collaborating Centre for Mental Health The British Psychological Sociaety \& Royal College of Psychiatrists; 2007.

18. Reilly N, Harris S, Loxton D, Chojenta C, Forder P, Milgrom J, et al. Disparities in reported psychosocial assessment across public and private maternity settings: a national survey of women in Australia. BMC Public Health. 2013;13.

19.Kohlhoff J, Hickinbotham R, Knox C, Roach V, Barnett B. Antenatal psychosocial assessment and depression screening in a private hospital. Australian and New Zealand Journal of Obstatrics and 
Gynaecology. 2016;56:173-8.

20.Greenwood J, Thorogood N. Qualitative methods for health research. 2nd ed. London: SAGE Publication; 2009.

21.Silverman D. Doing qualitative research. 3rd ed. London: Sage; 2010.

22.Grbich C. Qualititive data analysis: An introduction. London: SAGE Publications Ltd; 2013.

23.Doody O, Slevin E, Taggart L. Focus group interviews in nursing research: part 1. British Journal of Nursing. 2013;22(1):16-9.

24.Creswell J. Research Design Qualitative and Quantitative Approaches. California: Thousand Oaks; 1994.

25.Streubert H, Carpenter D. Qualitative research in nursing. China: Wolters Kluwer Health; 2011.

26.Kalra H, Reilly N, M-P. A. An evaluation of routine antenatal depression screening and psychosocial assessment in a regional private maternity setting in Australia. Aust NZ J Obstet and Gynaecol. 2018;58(1):1-7.

27.International Marce Society. Psychosocial assessment and depression screening in perinatal women position statement. Swanesa, Wales; 2014.

28.Austin M-P, Highet N, Expert Working Group. Mental Health Care in the Perinatal Period: Australian Clinical Practice Guideline. Melbourne Centre of Perinatal Excellence; 2017.

29.Jones CJ, Creedy DK, Gamble JA. Australian midwives' awareness and management of antenatal and postpartum depression. Women And Birth. 2011;183.

30.Cox JL, Holden JM. Detection of postnatal depression. Development of the 10-item Edinburgh postnatal depression scale. British Journal Of Psychiatry. 1987;150:782-6.

31.Boyd RC, Le HN, Somberg R. Review of screening instruments for postpartum depression. Archives of Women's Mental Health. 2005;8:141-53.

32.Matthey S, Agostini F. Using the Edinburgh Postnatal Depression Scale for women and men-some cautionary thoughts. Archives of Women's Mental Health. 2017;20(2):345-54.

33.Armstrong SJ, Small RE. The paradox of screening: rural woman's views on screening for postnatal depression. BMC Public Health. 2010;vol. 10.

34.Sims DJ, Fowler C. Postnatal psychosocial assessment and clinical decision-making, a descriptive study. Journal of Clinical Nursing. 2018;27(19-20):3739-49. 
35.Austin M-P, Committee TMSPSA. Marce International Society position statement on psychosocial assessment and depression screening in perinatal women. Best practice and research in obstetrics and gynaecology. 2013;28:179-87.

36.NSW Department of Health. Child and family health nursing professional practice framework 20112016. 2011.

37.Psaila K, Schmied V, Fowler C, Kruske S. Discontinuities between maternity and child and family health services: health professional's perceptions. BMC Health Services Research. 2014;14(1):1-22.

38.Rossiter C, Fowler C, Hesson A, Kruske S, Homer CSE, Kemp L, et al. Australian parents' experiences with universal child and family health services. Collegian. 2018.

39.Goodman J, Tyer-Viola L. Detection, treatment and referral of perinatal depression and anxiety by obstetric providers. Journal of women's health. 2010;19(3):477 - 90.

40.Yelland J, Brown S. Asking women about mental health and social adversity in pregnancy: Results of an Australian population-based survey. Birth Issues In Perinatal Care. 2014;41(1):79-89.

41.Mollart L, Newing C, Foureur M. Midwives emotional wellbeing: impact of conducting a structured antenatal psychosocial assessment (SAPSA). Women and Birth. 2009;22(3):82-8.

42.Reilly N, Harris S, Loxton D, Chojenta C, Forder P, Austin M-P. The impact of routine assessment of past or current mental health on help-seeking in the perinatal period. Women and Birth. 2014;27(4):e20-e7.

43.Reilly N, Yin C, Monterosso L, Bradshaw S, Neale K, Harrison B, et al. Identifying psychosocial risk among mothers in an Australian private maternity setting: a pilot study. Australia and New Zealand Journal of Obstetrics and Gynaecology. 2015;55:453-8.

44.Paulson J., S. B. Prenatal and postpartum depression in fathers and its association with maternal depression. Journal American Medical Association 2010;303(19):1961 - 8.

\section{Tables}




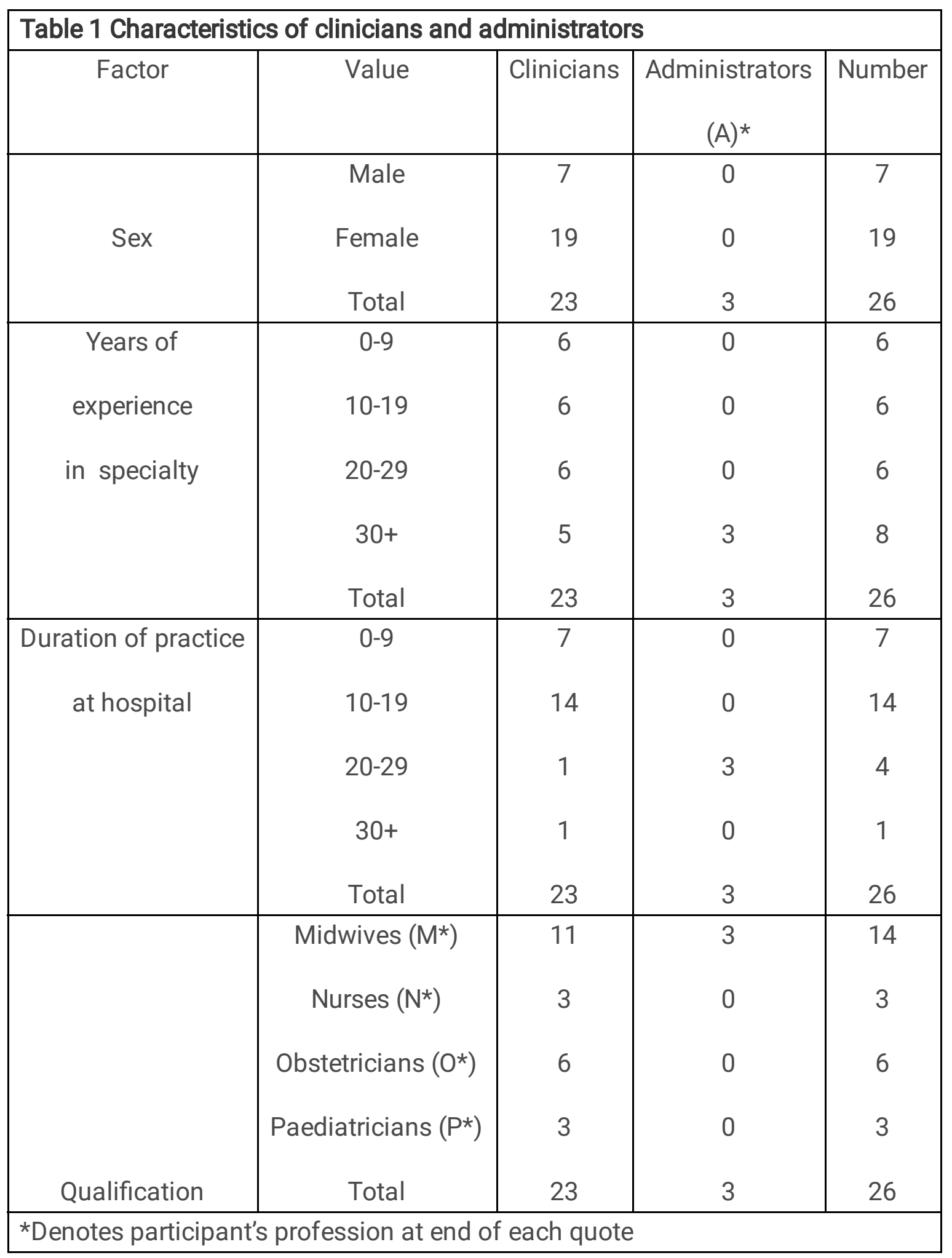




\begin{tabular}{|c|c|}
\hline Participant Pseudonym & Participant Professional Background \\
\hline Sarah & Manager \\
\hline Jessica & Accountant \\
\hline Rebecca & Teacher and Social Worker \\
\hline Melissa & Doctor \\
\hline Lauren & Hair Stylist \\
\hline Emma & Lawyer \\
\hline Nicole & Real Estate Broker \\
\hline Amanda & HR Director \\
\hline
\end{tabular}




\begin{tabular}{|l|l|}
\hline Box 1 - Recruitment Strategies & Strategy \\
\hline Participant & $\begin{array}{l}\text { Distribution of study announcement via email, by maternity } \\
\text { administrator } \\
\text { midwives and nursery nurses }\end{array}$ \\
& $\begin{array}{l}\text { Study posters placed in maternity clinician common areas } \\
\text { Invitation to attend an in-service education session on } \\
\text { Transition to Parenthood, provided by DS } \\
\text { Snowball recruitment was undertaken to recruit nurses and } \\
\text { midwives }\end{array}$ \\
\hline visiting medical officers (VMOs) - & $\begin{array}{l}\text { DS visited VMO consulting rooms to discuss the study with } \\
\text { clerical staff and provide a study flyer }\end{array}$ \\
& DS telephoned to follow-up with interested VMOs \\
& $\begin{array}{l}\text { Snowball recruitment was undertaken to recruit additional } \\
\text { VMOs }\end{array}$ \\
\hline women participants & $\begin{array}{l}\text { Recruited directly by the researcher at hospital prenatal } \\
\text { classes }\end{array}$ \\
\hline
\end{tabular}

\begin{tabular}{|l|l|}
\hline \multicolumn{2}{|l|}{ Box 2 - Themes and Subthemes on Assessment of Risk of Mental IIIness } \\
\hline Theme & Subtheme \\
\hline 1. Characteristics of assessment & Part of routine perinatal care \\
& Comprehensive psychosocial assessment \\
& Coordinated between care providers \\
\hline 2. Barriers to assessment & Lack of mental health resources \\
& Non-referral for mental health services \\
\hline
\end{tabular}

